\title{
GaN nanodiode arrays with improved design for zero-bias sub-THz detection
}

\author{
H Sánchez-Martín ${ }^{1}$, S Sánchez-Martín ${ }^{1}$, I Íñiguez-de-la-Torre ${ }^{1}{ }^{(1)}$, S Pérez $^{1}$ (),

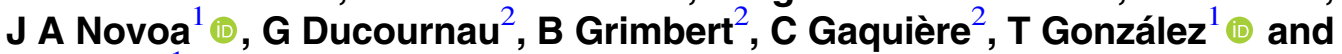 \\ J Mateos ${ }^{1}$ (1) \\ ${ }^{1}$ Departamento de Física Aplicada, Universidad de Salamanca, E-37008 Salamanca, Spain \\ ${ }^{2}$ Institut d'Electronique, de Microlectronique et de Nanotechnologie, University of Lille 1, F-59100 Lille, \\ France \\ E-mail: hectorsanchezmartin@usal.es
}

Received 15 May 2018, revised 19 July 2018

Accepted for publication 1 August 2018

Published 20 August 2018

\begin{abstract}
GaN based self-switching diodes (SSDs) have been fabricated for the first time on SiC substrate. They have been characterized as RF power detectors in a wide frequency range up to $220 \mathrm{GHz}$, showing a cutoff frequency of about $200 \mathrm{GHz}$. At low-frequency, RF measurements exhibit a square law detection with a responsivity that well agrees with the calculations performed by means of a quasi-static model based on the shape of the $I-V$ curve. Exploiting such a model, a simple DC characterization allows defining design rules for optimizing the practical operation of the diode arrays as RF power detectors. As strategy to improve the performance of SSDs operating as zero-bias detectors at room temperature, in terms of responsivity and noise equivalent power, we suggest: (i) the reduction of the channel width and (ii) the increase of the number of diodes in parallel in order to reduce the total device impedance to a value that coincides with 3 times that of the transmission line (or antenna) to which they are connected.
\end{abstract}

Keywords: THz detection, GaN, semiconductor nanodiodes, responsivity, noise equivalent power

(Some figures may appear in colour only in the online journal)

\section{Introduction}

The large range of potential applications of the $\mathrm{THz}$ range of the electromagnetic spectrum [1], specifically for imaging and spectroscopic analysis, is presently encouraging the research for new detector technologies with higher sensitivity and bandwidth. The review presented in [2] shows the recent progress of the different $\mathrm{THz}$ technologies. The self-switching diode (SSD) architecture has been explored as a possible candidate as $\mathrm{THz}$ device since 2003, when A M Song [3] claimed it as a promising device for mm-wave [4] and $\mathrm{THz}$ detection [5] and mixing [6]. Various materials systems have been studied for the fabrication of SSDs: InGaAs [7], GaAs [8], $\mathrm{ZnO}$ [9], organic compounds [10] and graphene [11]. The use of GaN for the channel of the SSD was initially proposed for emission purposes (by generating Gunn oscillations $[12,13])$, but revealed interesting capabilities for high power detection [14], with on-wafer responsivities up to $100 \mathrm{~V} / \mathrm{W}$ at
$0.3 \mathrm{THz}$ with a $280 \mathrm{pW} \mathrm{Hz}^{-1 / 2}$ noise equivalent power (NEP). In a free-space configuration, values of 2.0 and $0.3 \mathrm{~V} /$ $\mathrm{W}$ were obtained at 0.30 and $0.69 \mathrm{THz}$ [15]. However, there is still much room for improvement of the performances of GaN SSDs, for which a systematic study of the influence of the different design parameters has never been done.

Westlund et al derived an analytical model in $[16,17]$ to study zero-bias detection with InAs SSDs, and obtained the influence of geometrical and material parameters on the responsivity and NEP. These and other previous studies [14], focused on the improvement of the intrinsic performance of the SSDs, show that reducing the width of the channel improves their responsivity but increases the impedance mismatch with the access transmission line due to the larger impedance of the diode. In this paper we will focus on the optimization towards the implementation of practical detectors by improving such impedance matching following two different strategies: (i) fabricating several diodes in parallel 
and (ii) increasing the characteristic impedance of the transmission line. We will then provide robust guidelines for the optimization of both the responsivity and the NEP of SSDs not only in terms of their geometry, but also based on the impedance matching. In this way, these devices could be implemented as zero-bias detectors into multi pixel $\mathrm{THz}$ imaging systems.

$\mathrm{GaN}$ is not the optimal material for fabricating high frequency detectors, since high mobility semiconductors such as InGaAs, InAs or graphene would provide much higher cutoff frequencies. However, it has inherent advantages for being used as high power detector, where the responsivity is not the key figure of merit, but the power handling capability. Moreover, Monte Carlo simulations have shown the possibility of producing Gunn oscillations in GaN SSDs [13], so that both sub-THz wave sources and detectors could be combined to produce an integrated transceiver based on this architecture. Indeed, the choice of the $\mathrm{SiC}$ substrate for the fabrication of the devices was done in order to improve the thermal properties of the devices for achieving high power capabilities. The only present technology providing sub-THz power above the watt level are vacuum tubes, which reach even $\mathrm{kW}$ around $100 \mathrm{GHz}$ [18], but the growing interest in applications in the sub- $\mathrm{THz}$ range foretell that other technologies for the fabrication of powerful sources will appear in the near future. On the other hand, at present power measurements above the microwatt level can only be done with slow detectors based on thermal effects. Thus, the interest for fast high power sub-THz detectors based on the GaN SSD technology lies in the fact that they could be applied when a fast and precise measure of a powerful sub-mm wave signal is needed, for example for the implementation of real time active imaging systems, THz radars or ultrafast receivers for high data bitrate telecommunications.

In this study we show results of GaN SSDs working as detectors, fabricated for the first time on a $\mathrm{SiC}$ substrate, which have been characterized on-wafer in two different frequency bands up to $220 \mathrm{GHz}$. After presenting in section 2 the devices under test and the experimental setup, in section 3 we will explain a quasi-static model by means of which the low-frequency responsivity of the SSDs can be easily extracted from DC measurements. This method is confirmed to be able to reproduce the values of the experimental RF responsivity. Later on, in section 4 , the results of the strategies for reducing the impedance mismatch will be shown in terms of responsivity and NEP; and finally, in section 5, the main conclusions of this work will be drawn.

\section{Devices under test and experimental measurements}

The SSDs were fabricated on a $\mathrm{AlGaN} / \mathrm{GaN}$ heterostructure grown on a $\mathrm{SiC}$ substrate. The AlGaN barrier is $25 \mathrm{~nm}$ thick and has a $35 \% \mathrm{Al}$ content. The technological process for the fabrication of the SSDs is similar to the one presented in [14], using both dry etching and ion implantation to define the asymmetric shape of the channel. We have found a large

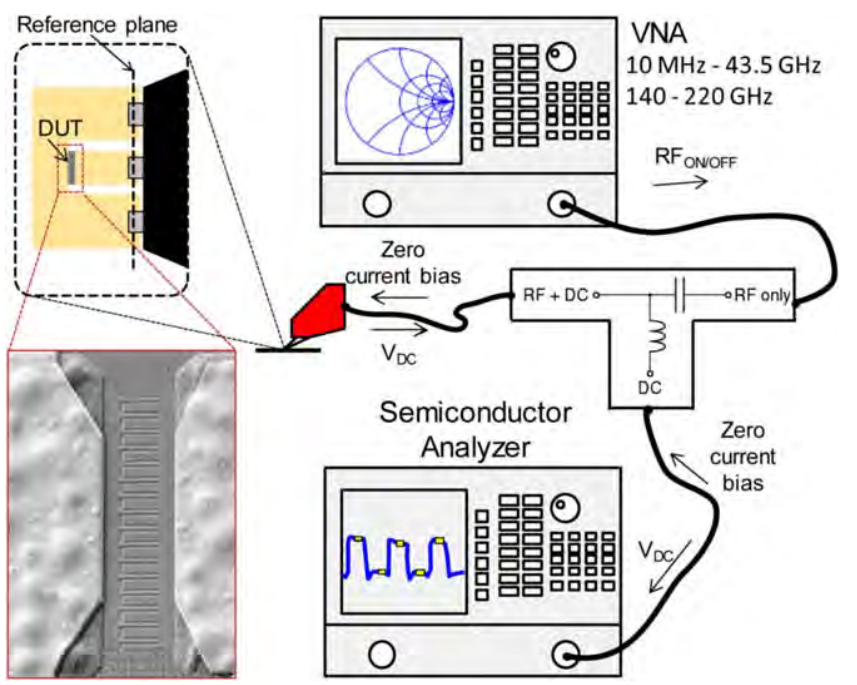

Figure 1. Schematic diagram of the microwave experimental setup. A probe station connects the devices by means of a bias-tee both to a vector network analyzer (VNA) providing the input RF power and to a semiconductor analyzer allowing to bias with zero current and measure the output voltage.

variability in the performance of nominally identical SSDs fabricated through ion implantation, presumably due to deviations on the width of the channel. Therefore, the results shown in this paper correspond to SSDs with etched trenches, which define channels with three different widths, (74, 100 and $200 \mathrm{~nm})$ and two lengths $(1.0$ and $2.0 \mu \mathrm{m})$. In addition to single diodes, arrays of 4,8 and 16 channels in parallel have also been fabricated.

The set-up presented in figure 1 has been used for the onwafer characterization of the RF detection capabilities of our devices. Measurements in the frequency ranges between $10 \mathrm{MHz}$ and $43.5 \mathrm{GHz}$, and between 140 and $220 \mathrm{GHz}(\mathrm{G}$ band) were carried out. Coplanar probes were used to contact the waveguide accesses of the SSDs in ground-signal-ground configuration to ensure a good microwave coupling. To estimate the power delivered to the DUT at the reference plane, the losses due to cables, connectors and probes were taken into account. A vector network analyzer (VNA) was used as RF power generator and, by means of a bias-tee, a semiconductor analyzer was used to bias the diode with zero current and to record the output voltage. The RF signal was chopped by switching on and off the output of the VNA, and the output voltage was determined by taking the difference in the DC voltage generated by the SSD array in presence and absence of the RF signal.

The responsivity, calculated as the ratio between the measured output voltage and the incident RF power, is shown in figure 2 as a function of the frequency for a SSD with one single channel of $L=2 \mu \mathrm{m}$ and $W=100 \mathrm{~nm}$, as an example. The measurements were performed in the two mentioned bands; the input power is $1 \mathrm{~mW}$ in the $0.01-43.5 \mathrm{GHz}$ band, while for the $140-220 \mathrm{GHz}$ band is between 1 and $10 \mu \mathrm{W}$. In the figure it can be observed that, in spite of the almost imperceptible nonlinearity of the $I-V$ curve of the device, values of tens of $\mathrm{V} / \mathrm{W}$ are obtained for the responsivity. The 


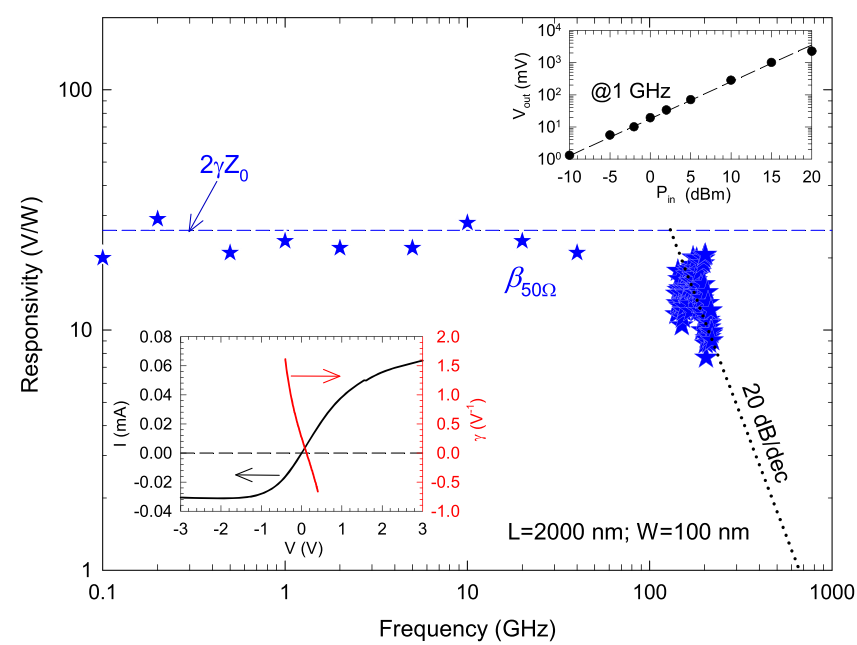

Figure 2. $\log -\log$ plot of the responsivity versus frequency of a SSD with just one channel with $L=2 \mu \mathrm{m}$ and $W=100 \mathrm{~nm}$. The dashed line shows the value extracted from the quasi-static model based on the DC measurements (in the strong mismatch limit, where it can be approximated by $2 \gamma Z_{0}$ ). The bottom inset shows the $I-V$ curve and the bowing coefficient $\gamma$. The top right inset displays the output voltage detected for a $1 \mathrm{GHz}$ signal, sweeping the injected power from -10 to $20 \mathrm{dBm}$. The dashed line shows a very good square law dependence.

parameter measuring the nonlinearity of the device is the bowing coefficient, $\gamma$, defined as $\frac{\mathrm{d}^{2} I}{\mathrm{~d} V^{2}} / \frac{\mathrm{d} I}{\mathrm{~d} V}$. The bottom inset of figure 2 shows that, for low bias voltages, $\gamma$ takes non-zero values high-enough to achieve the reported responsivity.

The response of this GaN SSD reveals a square law detection (output voltage proportional to the input power) up to around $20 \mathrm{dBm}$, the limit of the measurement setup, top panel inset of figure 2. This range of input power for which linear response is obtained can be further extended to much higher values by adding parallel channels and by using wider devices (with lower responsivity). Since these two strategies do not affect their frequency response, linear detection with SSDs at high power levels and high frequency can be envisaged. The high power handling capability at high frequencies is indeed one of the main features of the arrays of GaN SSDs proposed in this paper for being competitive with respect to the widely used GaAs Schottky barrier diode (SBD) and other $\mathrm{THz}$ technologies such as GaN lateral SBDs [19], low turn-on voltage InGaAs/InAlGaAs diodes [20] or backward diodes based on GaSb and GaAsSb [21, 22]. Indeed, even if the responsivity of SBDs is much higher than that of SSDs, their size has to be much decreased for $\mathrm{THz}$ applications in order to reduce the capacitance, thus limiting their input power level. Another detrimental consequence of the downscaling of SBDs is that the NEP is degraded, since it increases with the resistance of the devices. The SSD architecture allows overcoming this trend because their capacitance and resistance are not coupled: adding more channels in parallel reduces the total resistance but does not increase much the capacitance.

\section{Quasi-static model for RF measurements}

Following the quasi-static model described in [23], a generic nonlinear electronic device can be used for AC detection by exploiting the non-zero value of $\gamma$, that, for a one-port diode, can be obtained easily from its $I-V$ curve. The voltage responsivity of such device, $\beta_{\mathrm{opt}}$, defined as the rate between the open circuit output voltage and the input $\mathrm{AC}$ power, can then be calculated as:

$$
\beta_{\mathrm{opt}}=\frac{1}{2} R \gamma,
$$

$R=\mathrm{d} V / \mathrm{d} I$ being the resistance of the diode [23]. This is the optimum value of the voltage responsivity that such diode can provide, corresponding to perfect matching conditions for the input AC signal. In a practical case, part of the injected power is reflected due to the mismatch between the device and the transmission line. To estimate the reflected power, the reflection coefficient, $\Gamma=\left(R-Z_{0}\right) /\left(R+Z_{0}\right)$ must be considered, with $Z_{0}$ the characteristic impedance of the line, usually $50 \Omega$. Thus, the theoretical value to be compared with the measured responsivity when driving the diode with a $50 \Omega$ source, perfectly matched with the line, is given by:

$$
\beta_{50 \Omega}=\beta_{\mathrm{opt}}\left(1-|\Gamma|^{2}\right) \text {. }
$$

The good agreement between the experimental measurements of responsivity and the simple estimate of $\beta_{50 \Omega}$ given by equations (1) and (2), taking as a base the DC measurements of the $I-V$ curves of the diodes, was already demonstrated in [24] for SSDs and in [25] for SBDs.

If the SSD is sternly mismatched to the line $\left(R \gg Z_{0}\right)$, $\beta_{50 \Omega}$ takes the following value, independent of the diode resistance:

$$
\beta_{50 \Omega}=2 \gamma Z_{0} .
$$

The dashed line of figure 2 shows the value obtained with this quasi-static model, $26 \mathrm{~V} / \mathrm{W}$, which coincides with the measurements in the $0.01-43.5 \mathrm{GHz}$ band. In the G-band, a $20 \mathrm{~dB} / \mathrm{dec}$ frequency roll off is observed, with a cutoff frequency of around $200 \mathrm{GHz}$.

This quasi-static model for the extraction of the RF responsivity is able to describe the behavior of all the diodes within the uncertainty of the RF measurements, which, as shown in figure 2, is quite high. As a consequence, and in order to simplify the characterization process, we will use the quasi-static model to understand and predict the dependencies of the performance of the whole set of devices on several geometrical parameters, based just on their simple DC characterization, as done in [25] for SBDs. Figure 3 shows the resistance of SSDs with a single channel, denoted as $R_{0}$, as a function of the channel width. The channel length is $L=1 \mu \mathrm{m}$. As observed, $R_{0}$ is inversely proportional to the effective channel width, defined as $W_{\text {eff }}=W-2 W_{\mathrm{d}}$, with $W_{\mathrm{d}}$ the lateral depletion due to the surface charges at the sidewalls of the channel. The intersection of the $1 / R_{0}$ straight line with the $x$-axis provides the value of $2 W_{\mathrm{d}}$, which is around $53 \mathrm{~nm}$, similar to that found in previously fabricated SSDs [14]. As expected from the model proposed in [11], in which SSDs are 


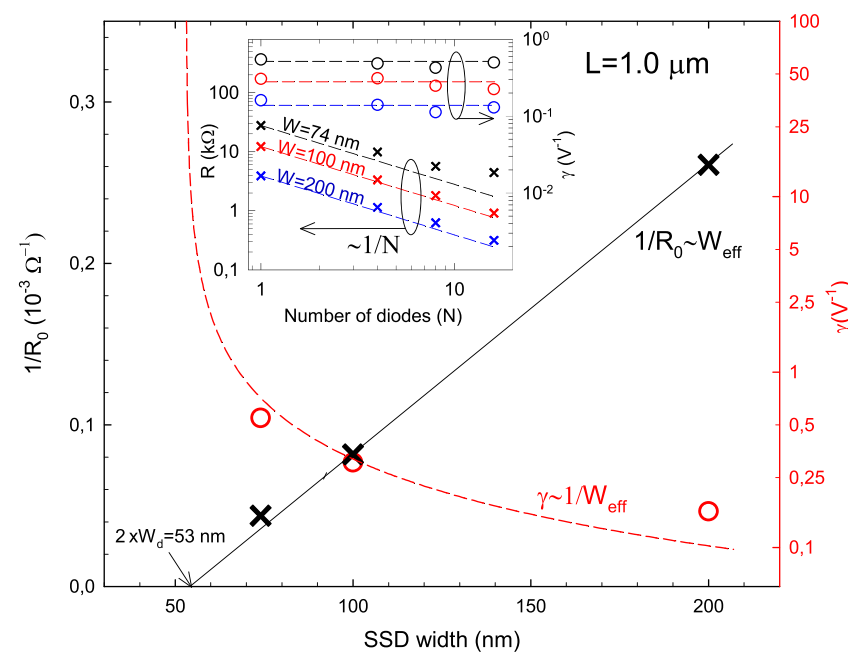

Figure 3. Admittance of single channel SSDs, $1 / R_{0}$ (crosses, left axis), and bowing coefficient, $\gamma$ (circles, right axis in log scale) extracted form the measured $I-V$ curve of the SSDs with $L=1 \mu \mathrm{m}$ as a function of the channel width. The inset shows in log-log scale the resistance (crosses, left axis) and $\gamma$ (circles, right axis) as a function of the number of diodes, the dashed lines are eye guides with $1 / N$ and constant behavior, respectively.

represented as ideal MOSFETs with gate and drain shorted (in the triode-region), the resistance and the bowing coefficient approximately scale as $1 / W_{\text {eff }}$.

As a consequence the matched responsivity, $\beta_{\text {opt }}$, should go as $1 / W_{\mathrm{eff}}^{2}$, and this expected trend is roughly followed by the fabricated devices, as shown in figure 4(a). As the impedances of the devices are well two orders of magnitude higher than the $50 \Omega$ of the line, the unmatched responsivity, $\beta_{50 \Omega}$, is only function of $\gamma$ (see equation (3)) and should therefore follow a $1 / W_{\text {eff }}$ dependence. Such a dependence is also represented in figure 4(a), where it is found not to be far from the trend exhibited by the experimental results obtained in two ways, from the quasi-static model and by means of a direct measure at $1 \mathrm{GHz}$, which show similar values within their respective uncertainties.

Even if the responsivity of a detector is an important quantity for characterizing its performance, the most important parameter is its sensitivity, the minimum power it can detect. The NEP, defined as the input power that provides an output voltage that coincides with the voltage noise of the detector per square root bandwidth, is used to measure the sensitivity. This figure of merit, which quantifies the weakest detectable signal, can be calculated at zero bias assuming that the detector generates just thermal noise. Thus, we obtain it as the ratio of the electronic Johnson noise, given by the Nyquist theorem, and the responsivity:

$$
\mathrm{NEP}_{\text {opt }, 50 \Omega}=\frac{\sqrt{4 k_{\mathrm{B}} T R}}{\beta_{\mathrm{opt}, 50 \Omega}},
$$

with $k_{\mathrm{B}}$ the Boltzmann constant and $T$ the temperature (in both matched and unmatched conditions, that we will call in the following $\mathrm{NEP}_{\text {opt }}$ and $\mathrm{NEP}_{50 \Omega}$, respectively).

Applying the ideal MOSFET model of [11], $\mathrm{NEP}_{\text {opt }}$ should go as $W_{\mathrm{eff}}^{3 / 2}$ and $\mathrm{NEP}_{50 \Omega}$ as $W_{\mathrm{eff}}^{1 / 2}$ (if the DUT is

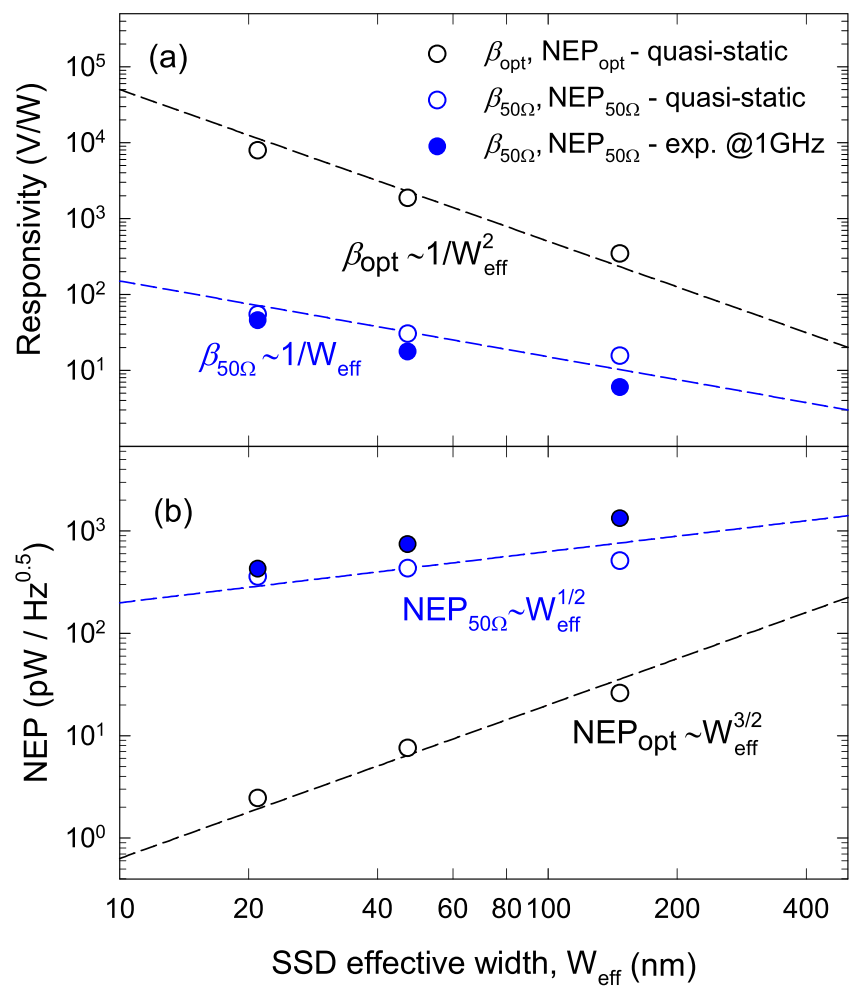

Figure 4. Log-log plot of the (a) responsivity and (b) NEP versus the effective channel width extracted with the quasi-static model from DC measurements (void symbols) and experimental measurements @ $1 \mathrm{GHz}$ (solid symbols) of SSDs with $N=1$ and $L=1 \mu \mathrm{m}$. The optimum and $50 \Omega$ values are plotted in both cases, obtained using the equations (1), (2) and (4). Dashed lines indicate the trends of the different magnitudes expected in the model of [11].

severely mismatched to the line). Figure 4(b) shows these tendencies compared with RF measurements done at $1 \mathrm{GHz}$ and the estimation obtained from the quasi-static model. Even if they do not show a perfect agreement, as in the case of figure 3 , the indicated tendencies predict roughly well the experimental results. These performances of SSDs fabricated with $\mathrm{GaN}$ on $\mathrm{SiC}$ substrate are similar to those previously obtained on $\mathrm{Si}$ substrate [14, 15], as expected, since heat dissipation is not an issue when operating as zero-bias detectors. The advantages of the SiC substrate would become visible at high input power levels, when self-heating effects arise.

\section{Optimizing impedance matching}

Taking advantage of the easily parallelizable architecture of SSDs, the impedance matching can be improved by using an array of diodes in parallel, which also benefits the signal-tonoise ratio [5] and therefore decreases the value of the NEP. The inset of figure 3 shows how the measured resistance is reduced in a factor $N$, except for the case $W=74 \mathrm{~nm}$, in which the scaling is not perfect, probably due to the variability of the fabrication process, at the limit of the resolution of the technology. Interestingly, the value of the bowing coefficient $\gamma$ is constant with $N$, thus allowing to improve the 
impedance matching without losing nonlinearity. Regarding the responsivity, its optimum value is obtained for $N=1$, since all the input power goes to just one channel. Following equation (1), the parallelization reduces the optimum responsivity as $1 / N$, so that $\beta_{\mathrm{opt}}=\frac{1}{2} \frac{R_{0}}{N} \gamma$. On the other hand, when the impedance matching is improved, using equation (2) one can find that the measured responsivity has the following dependence on $N$ :

$$
\beta_{50 \Omega}=2 \gamma \frac{R_{0}^{2} Z_{0}}{\left(R_{0}+N Z_{0}\right)^{2}} .
$$

Since, as observed in the inset of figure 3, the value of $R_{0}$ is at least several $\mathrm{k} \Omega, R_{0} \gg Z_{0}$ in all cases and the maximum value of $\beta_{50 \Omega}$ is obtained when $N$ is low and the SSDs resistance still fulfills the condition $R=R_{0} / N \gg Z_{0}$. As a consequence, in this range of $N$, the value of $\beta_{50 \Omega}$ remains almost constant, taking the value predicted by equation (3), $2 \gamma Z_{0}$. This trend is observed both in the results obtained with the quasi-static model and those directly measured at $1 \mathrm{GHz}$, with values that well agree between them. When $N$ is further increased and the impedance matching improves, $\beta_{50 \Omega}$ decreases as $1 / N$, dropping to one fourth of its maximum value when $R=Z_{0}$ (and $\beta_{50 \Omega}=\beta_{\text {opt }}$ ). For even higher values of $N, \beta_{50 \Omega}$ falls more rapidly, reaching a $1 / N^{2}$ dependence when $R \ll Z_{0}$.

The predictions of equations (1) and (5) are satisfactorily compared in figure 5(a) with the experimental results for SSDs with $L=1 \mu \mathrm{m}$ and $W=100 \mathrm{~nm}$. In this case, due to the high value of $R_{0}$, above $10 \mathrm{k} \Omega, \beta_{50 \Omega}$ remains in the range where it is almost independent of $N$, even for the highest number of parallel diodes fabricated for this geometry, $N=16$. In order to distinguish the range of improved impedance matching, we also show in the figure the results for diodes with much lower resistance (but also much lower nonlinearity), called V-shaped diodes, initially designed for triggering the onset of Gunn oscillations [13]. Despite the lower value of $\beta_{50 \Omega}$ (due to a small $\gamma$ ), its lower impedance $\left(R_{0}\right.$ is around $2 \mathrm{k} \Omega$ ) and the higher number of parallel channels (arrays of 64 SSDs were fabricated) allow to decrease the resistance below that of the $50 \Omega$ waveguide. In this way, we enter into the range where the responsivity falls with $N$, again well following the dependence described by equation (5).

Concerning the NEP, the dependence on $N$ can be obtained inserting in equation (4) the values of the optimum and $50 \Omega$ responsivities given by equations (1) and (5). Even if $\mathrm{NEP}_{\text {opt }}$ monotonically increases as $N^{1 / 2}$, apparently showing a degradation of the noise behavior when $N$ increases, the value of $\mathrm{NEP}_{50 \Omega}$ has three different regimes. It starts with a decay as $1 / N^{1 / 2}$ for low $N$, going to a slow increase as $N^{1 / 2}$ when $R \sim Z_{0}$, and finishing with a faster degradation as $N^{3 / 2}$ for $R \ll Z_{0}$.

The most interesting observation is that the minimum of $\mathrm{NEP}_{50 \Omega}$ is not reached when a perfect impedance matching is obtained, but when the condition $R=3 Z_{0}$ is fulfilled (i.e. for $\left.N=R_{0} / 3 Z_{0}\right)$. However, when $N$ takes this particular value, corresponding to an optimum signal to noise ratio, the measured responsivity falls to $9 / 16(56 \%)$ of its maximum,

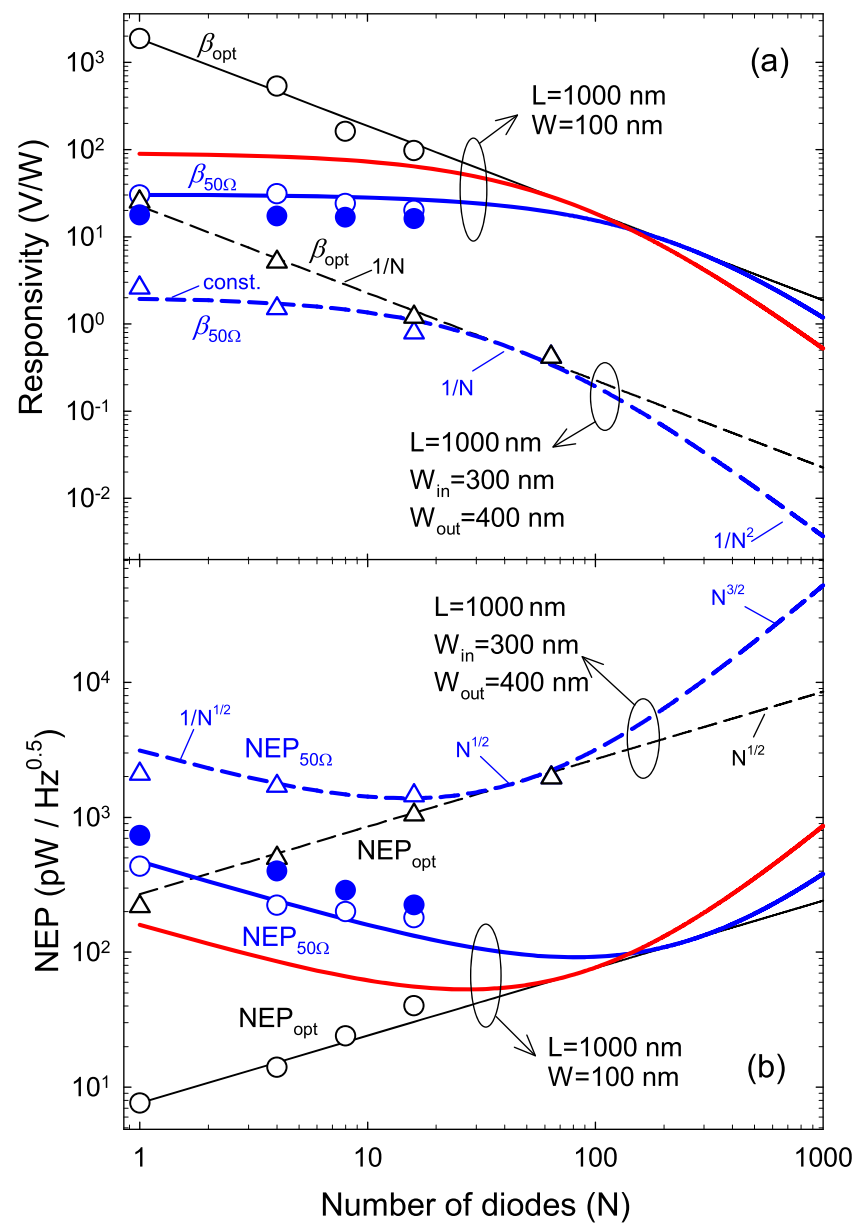

Figure 5. Log-log plot of (a) responsivity and (b) NEP versus the number of channels extracted with the quasi-static model (void symbols) and experimental measurements@1 GHz (solid symbols) of SSDs with $L=1 \mu \mathrm{m}$ and $W=100 \mathrm{~nm}$. The optimum and $50 \Omega$ values are plotted in both cases. The results for a much wider V-shape channel sketched in the graph (with $L=1 \mu \mathrm{m}$, $W_{\text {in }}=300 \mathrm{~nm}$ and $W_{\text {out }}=400 \mathrm{~nm}$, achieving a better matching to the $50 \Omega$ line) are also shown for comparison. The lines indicate the trends of the different quantities following equations (1), (4) and (5) with $Z_{0}=50 \Omega$. The red lines show the analytical calculations using $Z_{0}=150 \Omega$

$\beta_{50 \Omega}=(9 / 8) \gamma Z_{0}$. For example, the SSD with $L=1 \mu \mathrm{m}$ and $W=100 \mathrm{~nm}$ (with $R_{0}=12 \mathrm{k} \Omega$ ) would provide the best performance in a $50 \Omega$ system by integrating approximately 80 diodes in parallel. In this configuration, the values of the responsivity and NEP would be $\beta_{50 \Omega}=17 \mathrm{~V} / \mathrm{W}$ and $\mathrm{NEP}_{50 \Omega}=92 \mathrm{pW} \mathrm{Hz}^{-1 / 2}$, as compared to $30 \mathrm{~V} / \mathrm{W}$ and $425 \mathrm{pW} \mathrm{Hz}^{-1 / 2}$, respectively, obtained for $N=1$. As we can see, these optimum matching conditions attained by correctly choosing the value of $N$ at the time of fabricating the devices, allow for an important decrease of the NEP (a 4.6 factor), with just a slight degradation of the responsivity (1.8 factor) with respect to a single-channel SSD. This behavior is analogous to that of transistors, in which the optimum bias for high gain does not coincide with that for low noise operation.

Another way to improve the impedance matching of SSDs is by connecting them to a high-impedance coplanar waveguide. For example, by reducing the signal line to $1 \mu \mathrm{m}$ 
and increasing the ground plane spacing to $100 \mu \mathrm{m}$, the characteristic impedance of the CPW can be increased to $Z_{0}=150 \Omega$. In that case, an adequately tapered transition to the present $50 \Omega$ accesses (with a $30 \mu \mathrm{m}$ line and $20 \mu \mathrm{m}$ spacing) has to be implemented in order to keep the reflection coefficient as low as possible. The values of $\beta_{50 \Omega}$ and $\mathrm{NEP}_{50 \Omega}$ calculated using $Z_{0}=150 \Omega$, plotted also in figure 5 , are much improved with respect to the case of $Z_{0}=50 \Omega$. Indeed, a $3 \times$ factor in the responsivity and $\sqrt{ } 3 \times$ in the NEP (with values of around $50 \mathrm{~V} / \mathrm{W}$ and $53 \mathrm{pW} \mathrm{Hz}^{-1 / 2}$, respectively) could be obtained with a lower number of parallel channels $(3 \times$ less, around 27$)$. In that case the problem would be that the small size of the signal line would force the use of improved access designs to accommodate such number of devices, that needs around a $30 \mu \mathrm{m}$ contour.

\section{Conclusions}

SSDs with GaN channels fabricated on $\mathrm{SiC}$ substrate were explored extensively as zero-bias detectors, and were confirmed to provide similar performances as those fabricated on $\mathrm{Si}$ substrate. First, for a diode with $L=2 \mu \mathrm{m}$ and $W=100 \mathrm{~nm}$, a responsivity exhibiting a cutoff frequency of about $200 \mathrm{GHz}$ was observed, along with a square law response up to $20 \mathrm{dBm}$ of input power. Then, responsivities calculated with a quasi-static model based on DC measurements were confirmed to correctly reproduce the RF experiments. This quasi-static estimation of the responsivity constitutes a robust tool for the further optimization of the main figures of merit of the SSDs operating as detectors for, e.g., THz imaging applications. The key result obtained is that the optimum detector performance (in terms of minimum NEP) is achieved with an array of SSDs providing a resistance 3 times that of the characteristic impedance of the transmission line to which it is connected. In these conditions the responsivity decreases to $9 / 16$ of its maximum value (obtained for $N=1$ ). The use of a high-impedance transmission line has also been explored as a useful way of improving the detector performances.

\section{Acknowledgments}

This work has been partially supported by the Spanish MINECO through project TEC2017-83910-R and by the Consejería de Educación de la Junta de Castilla y León through project SA022U16.

\section{ORCID iDs}

I Íñiguez-de-la-Torre (iD https://orcid.org/0000-00022059-0955

S Pérez (ib https://orcid.org/0000-0002-1494-0795
J A Novoa (1) https://orcid.org/0000-0003-4229-8928

T González (i) https://orcid.org/0000-0002-3706-3211

J Mateos (1) https://orcid.org/0000-0003-4041-7145

\section{References}

[1] Mittleman D M 2003 Sensing with THz Radiation (Berlin: Springer)

[2] Dhillon S S et al 2017 J. Phys. D: Appl. Phys. 50043001

[3] Song A M, Missous M, Omling P, Peaker A R, Samuelson L and Seifert W 2003 Appl. Phys. Lett. 831881

[4] Balocco C et al 2005 Nano Lett. 51423

[5] Balocco C, Halsall M, Vinh N Q and Song A M 2008 J. Phys.: Condens. Matter 20384203

[6] Torres J et al 2013 Semicond. Sci. Technol. 28125024

[7] Balocco C, Kasjoo S R, Zhang L Q, Alimi Y and Song A M 2011 Appl. Phys. Lett. 99113511

[8] Balocco C, Kasjoo S R, Lu X F, Zhang L Q, Alimi Y, Winnerl S and Song A M 2011 Appl. Phys. Lett. 98223501

[9] Irshaid M Y, Balocco C, Luo Y, Bao P, Brox-Nilsen C and Song A M 2011 Appl. Phys. Lett. 99092101

[10] Kettle J, Whitelegg S, Song A M, Madec M B, Yeates S, Turner M L, Kotacka L and Kolarik V 2009 J. Vac. Sci. Technol. B 272801

[11] Westlund A, Winters M, Ivanov I G, Hassan J, Nilsson P A, Janzén E, Rorsman N and Grahn J 2015 App. Phys. Lett. 106 093116

[12] Iñiguez-de-la-Torre A, Iñiguez-de-la-Torre I, Mateos J, González T, Sangaré P, Faucher M, Grimbert B, Brandli V, Ducournau G and Gaquière C 2012 J. Appl. Phys. 111 113705

[13] Millithaler J F, Iñiguez-de-la-Torre I, Iñiguez-de-la-Torre A, González T, Sangaré P, Ducournau G, Gaquière C and Mateos J 2014 Appl. Phys. Lett. 104073509

[14] Sangaré P et al 2013 J. Appl. Phys. 113034305

[15] Daher C, Torres J, Iñiguez-de-la-Torre I, Nouvel P, Varani L, Sangaré P, Ducournau G, Gaquière C, Mateos J and González T 2016 IEEE Trans. Electron Devices 63353

[16] Westlund A et al 2015 Solid-State Electron. 10479

[17] Westlund A, Sangaré P, Ducournau G, Nilsson P-Å, Gaquière C, Desplanque L, Wallart X and Grahn J 2013 Appl. Phys. Lett. 103133504

[18] He W, Donaldson C R, Zhang L, Ronald K, McElhinney P and Cross A W 2013 Phys. Rev. Lett. 110165101

[19] Cywinski G, Yahniuk I, Szkudlarek K, Kruszewski P, Yatsunenko S, Muzioł G, Skierbiszewski C, But D and Knap W 20162016 MIXDES-23rd Int. Conf. Mixed Design of Integrated Circuits and Systems vol 346

[20] Radisic V, Leong K, Zhang C, Loi K K and Sarkozy S 2013 IEEE Trans. Microw. Theory Tech. 612949

[21] Zhang Z, Rajavel R, Deelman P and Fay P 2011 IEEE Microw. Wirel. Compon. Lett. 21267

[22] Takahashi T, Sato M, Hirose T and Hara N 2010 Japan. J. Appl. Phys. 49104101

[23] Cowley A M and Sorensen H O 1966 IEEE Trans. Microw. Theory Tech. 14588

[24] Iñiguez-de-la-Torre I et al 2014 IEEE Trans. Terahertz Sci. Tech. 4670

[25] Han R, Zhang Y, Coquillat D, Videlier H, Knap W, Brown E and O K K 2011 IEEE J. Solid-State Circuits 462602 